\title{
Optical Projection Tomography of Vertebrate Embryo Development
}

\author{
Laura Quintana and James Sharpe
}

\section{INTRODUCTION}

This article includes a brief introduction to optical projection tomography (OPT) as a method for imaging whole vertebrate embryos. To place OPT in its proper context within the field of three-dimensional (3D) embryo imaging, the article starts with a brief overview of the previously existing techniques, both destructive and nondestructive. Next is a description of how OPT works and its advantages over other approaches. Two practical sections are then presented that consider the applications of OPT and how to get the best results from OPT in terms of specimen preparation and imaging modes.

\section{RELATED INFORMATION}

Information is available on High-Speed Optical Coherence Tomography Imaging of the Beating Avian Embryonic Heart (Gu et al. 2011a) and MRI in Developmental Biology and the Construction of Developmental Atlases (Ruffins and Jacobs 2011). Protocols are available for Preparation of Mouse Embryos for Optical Projection Tomography Imaging (Quintana and Sharpe 2011) and Optical Coherence Tomography Imaging of Early Quail Embryos (Gu et al. 2011b).

\section{PREEXISTING TECHNIQUES FOR IMAGING WHOLE EMBRYOS}

\section{Physical Sectioning}

Until recently, the most common technique for recreating a 3D representation of a small biological specimen has been to physically cut it into hundreds of sections, photograph each one, and then recombine the images in a computer (Davidson and Baldock 2001). Although time-consuming, this approach has the advantage that any type of optical staining may be used because all that is needed is a series of digital images. Unfortunately, however, when a specimen is cut into thin sections, the data become essentially two-dimensional (2D). This means that information about the alignment of each section to its neighbor (the third dimension) is lost. In addition, for many common types of sectioning (e.g., microtome and cryosections), the embedding material (e.g., paraffin wax and optimal cutting temperature [OCT] compound) is not rigid enough to prevent the tissue from stretching and deforming when it is mounted onto glass slides. These two factors prevent reconstruction of the true 3D shape of the specimen from sections alone. For some experiments, this does not matter, but for tasks such as phenotyping it can be important to use a technique, such as OPT, that preserves true shape.

New sectioning techniques have been developed to speed up the process and overcome the "trueshape" problem—for example, block-face imaging (e.g., episcopic fluorescence image capture [EFIC] and high-resolution episcopic microscopy [HREM]) (see Weninger and Mohun 2002; Mohun and Weninger 2011). Instead of photographing each section after it has been cut and mounted on a glass slide, the section is imaged just before it is cut-at the surface of the block. If the positions of the camera and block are carefully controlled such that the focal plane always coincides with the cut surface of the block, the problems of both section deformation and alignment are simultaneously overcome. This approach can generate very high-resolution reconstructions of large specimens with excellent shape preservation. EFIC and especially the more recent HREM also allow colored stains to be analyzed (as with normal paraffin wax sections). This is very important for gene expression analysis because the most widespread protocol for detection of mRNAs involves the enzymatic conversion of BCIP/NBT (5-bromo-4-chloro-3-indolyl

Adapted from Imaging in Developmental Biology (ed. Sharpe and

Wong). CSHL Press, Cold Spring Harbor, NY, USA, 2011.

Cite as: Cold Spring Harb Protoc; 2011; doi:10.1101/pdb.top116

www.cshprotocols.org 
phosphate/4-nitro blue tetrazolium) to a purple precipitate (Hammond et al. 1998). However, so far the reliance on a colored precipitate means that multichannel labeling (e.g., with fluorescent antibodies) is not yet possible.

\section{Nondestructive Imaging}

The following nondestructive imaging techniques can be used to generate images from living embryos (OPT has yet to be optimized for this purpose); however, none of the techniques is ideal for analyzing 3D gene-expression-fixed embryos.

\section{$X$-Ray Micro-Computed Tomography $(\mu \mathrm{CT})$}

X-ray micro-computed tomography $(\mu \mathrm{CT})$ is well suited to specimens $\sim 1 \mathrm{~cm}$ in size and displays a resolution that is in principle ideal for embryos $(<10 \mu \mathrm{m})$. Metscher (2011) describes excellent examples of this approach. However, $\mu \mathrm{CT}$ is a nonoptical technique and thus cannot take advantage of fluorescently labeled antibodies. Moreover, the technique cannot be used to image a BCIP/NBT in situ pattern because $\mathrm{X}$ rays are not significantly absorbed by colored stains.

\section{Microscopic Magnetic Resonance Imaging ( $\mu M R I)$}

Microscopic magnetic resonance imaging ( $\mu \mathrm{MRI}$ ) has a potential advantage over $\mu \mathrm{CT}$ in that it can detect useful contrast between the tissues of untreated embryos (Dhenain et al. 2001), and it is currently a very useful approach for phenotyping and building atlases of embryo development (see Bamforth et al. 2011; MRI in Developmental Biology and the Construction of Developmental Atlases [Ruffins and Jacobs 2011]). However, the technology is generally very expensive, and resolution is significantly lower than that achieved by $\mu$ CT ( $25 \mu \mathrm{m}$ in the studies by Schneider et al. 2003). With respect to OPT, its main drawback is the paucity of assays for molecule-specific tissue labeling compared to optical techniques. Arguably, the most important potential future application for $\mu \mathrm{MRI}$ will be its ability to image dynamically changing $3 \mathrm{D}$ expression patterns in living embryos using high-contrast agents (Louie et al. 2000).

\section{Confocal Microscopy and Multiphoton Microscopy}

3D optical sectioning approaches have many advantages over nonoptical techniques (Potter et al. 1996). In particular, there is now available a veritable battery of fluorescent dyes that can be used in conjunction with a wealth of techniques to label biologically significant molecular distributions. At the cellular and cell-cluster levels, confocal microscopy generates impressive 3D results and is invaluable for cell biology. However, when it comes to analyzing tissues more than a few hundred micrometers in depth, confocal microscopy fails to image the entire intact specimen. In addition, another drawback to confocal and multiphoton approaches that is sometimes overlooked is their inability to generate $3 \mathrm{D}$ reconstructions of colored (absorption-based) staining patterns (as opposed to fluorescence patterns). There are many common assays for which the most reliable signal-detection method is still the production of a dark-colored precipitate (e.g., the use of BCIP/NBT to visualize whole-mount in situ hybridization). Although such precipitates can be detected easily on physically cut sections by brightfield microscopy, this particular assay for gene expression patterns cannot be imaged in 3D by fluorescence microscopy.

\section{Optical Coherence Tomography (OCT)}

Optical coherence tomography (OCT) has been used in a few developmental studies (see Yelbuz et al. 2002; High-Speed Optical Coherence Tomography Imaging of the Beating Avian Embryonic Heart [Gu et al. 2011a]; Optical Coherence Tomography Imaging of Early Quail Embryos [Gu et al. 2011b]; Larina et al. 2011), and it is the optical equivalent of ultrasound tomography: photons are sent into the specimen in one direction, and those reflected by the tissue are detected as they emerge. Their time of flight is calculated using interferometry. OCT can be used to image living tissue and can do so down to a depth of 2-3 mm; however, because of its reliance on interferometry, this approach is more limited than confocal microscopy in terms of signal detection. Because the light reflected from the specimen must be coherent with a reference beam, it is not possible to use fluorescence-based assays to detect gene expression patterns. 


\section{Microscopic Ultrasound Imaging}

Real microscopic ultrasound imaging is actually better suited to whole-embryo imaging than OCT, as it displays far greater depth penetration (it can image an entire mouse embryo, alive, within the uterus of a pregnant female) (see Foster and Brown 2011). Again, its strength lies in its ability to image living embryos, and, in this case, more convenient access to embryos within a pregnant mouse compared to MRI. However, for fixed specimens, this approach does not provide high resolution (achieving voxels $50 \mu \mathrm{m}$ across), nor does it feature convenient gene expression assays.

\section{HOW OPT WORKS}

The optical 3D microscopy techniques mentioned above (confocal microscopy, multiphoton microscopy, and optical coherence tomography) use a geometric arrangement that can be called section tomography. This essentially means that they sample the properties of a specimen at discrete points in space (confocal microscopy samples the fluorescence of a specimen, whereas OCT samples reflectiveness). In these cases, each measurement has explicit $x-y-z$ coordinates and can be mapped directly to a voxel in the resulting reconstruction. In contrast, imaging techniques that use the principle of projection tomography (often called "computed tomography") collect data that sum a measured property along a linear projection that traverses the entire specimen (see Kak and Slaney 1988). These projection data, which are gathered from a series of orientations through the specimen, do not directly represent voxels and must be transformed in order to recover the original structure of the specimen (using a filtered back-projection algorithm). Projection tomography is widely used to generate highresolution reconstructions in techniques that use $\mathrm{X}$ rays, $\gamma$ rays, or electron beams, because these rays are not deflected as they pass through their respective specimens (Massoud and Gambhir 2003; Midgley and Weyland 2003). A related form of tomography has also been applied to optical systems in the medical technique known as diffuse optical tomography (DOT) (for review, see Ntziachristos and Chance 2001). However, light rays are very prone to scattering as they pass through biological tissue, so DOT uses algorithms different from those of OPT, which attempt to compensate for these distortions and can produce only low-resolution reconstructions.

The approach adopted in OPT (Sharpe et al. 2002) is to suspend the specimen in an index-matching liquid to (1) reduce the scattering of light, and (2) reduce heterogeneities of refractive index throughout the specimen. This means that light passes through the specimen in more or less straight lines and the back-projection algorithm can, therefore, generate relatively high-resolution images. The liquid most often used is BABB or Murray's clear solution (a mixture of benzyl alcohol and benzyl benzoate). Inside the OPT scanning device, the specimen is maintained within the liquid and rotated through a series of angular positions ( $<1^{\circ}$ apart in our current design), and an image is captured at each orientation. The apparatus is carefully aligned to ensure that the axis of rotation is perpendicular to the optical axis, so that projection data pertaining to each plane are collected by a linear row of pixels on the chargecoupled device (CCD) of the camera (Fig. 1).

There are two imaging modes for OPT (for a fuller technical description, see Sharpe 2004). The first is transmission imaging or bright-field OPT. This mode corresponds closely to the method used by an X-ray CT scanner; that is, the specimen is illuminated from the side opposite the detector, and light rays (from a mercury lamp, or similar) pass through the specimen and are absorbed by varying amounts. The CCD camera records an image similar to a quantitative shadow of the specimen (the major difference being that OPT uses lenses to focus an image, whereas CT captures a genuine shadow).

The second mode is emission tomography or fluorescence OPT. As before, owing to the use of light and the consequent ability to use lenses to create focused images, this form of emission tomography is very different from a technique like positron emission tomography (PET). In fluorescence OPT, the entire specimen is illuminated with a specific wavelength (filtered from a mercury source), and fluorescently emitted light is then focused into an image on the CCD. Usually, the excitation is provided on the same side as the detection.

In both modes of OPT, the fact that lenses are used to focus an image means that OPT suffers one technical drawback compared to its X-ray equivalent: As with all optical imaging systems, there is only a limited depth of focus, and this usually cannot encompass the entire specimen. OPT, therefore, generally takes advantage of a compromise, which yields good results - the focal plane is positioned halfway between the axis of rotation and the edge of the specimen closest to the lenses. This maximizes the 
A

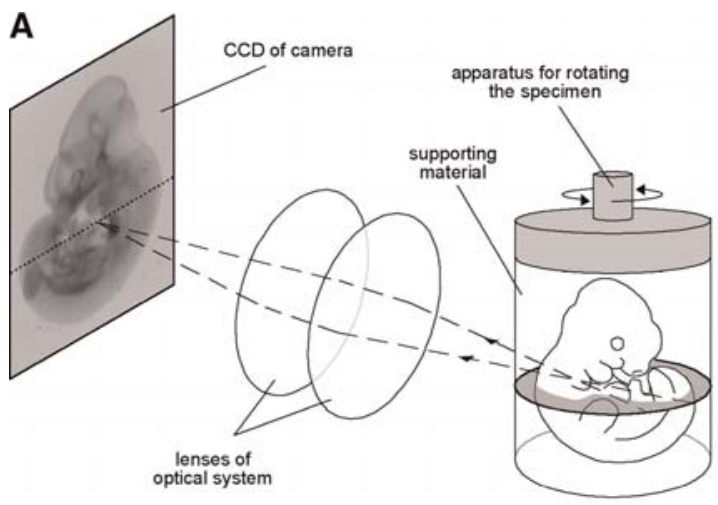

B

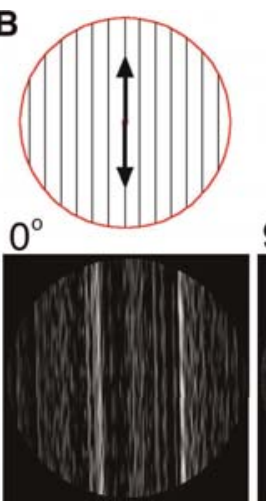

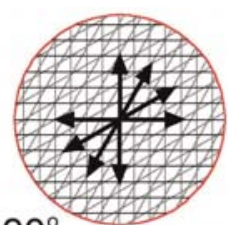
$90^{\circ}$

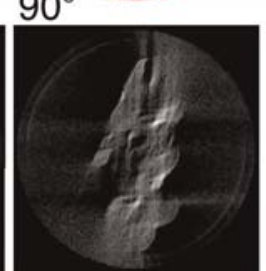

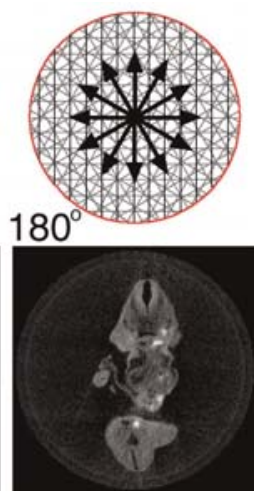

FIGURE 1. How OPT works. (A) Apparatus for obtaining OPT images. Light from the specimen (dashed lines) is focused to create an image on the CCD of the camera. The apparatus is aligned such that projections through a section perpendicular to the axis of rotation (gray oval) are focused onto a single row of pixels (dotted line). (B) As projections from successive angles $\left(0^{\circ}, 90^{\circ}, 180^{\circ}\right)$ are accumulated in the backprojection algorithm, a representation of the section is gradually reconstructed. (Top) Accumulated orientations of projection; (bottom) emerging reconstruction during the process. Although techniques such as X-ray CT require only $180^{\circ}$ of information, OPT benefits from a full $360^{\circ}$ rotation because the limited depth of focus means that views from opposite orientations do not contain the same information.

focused information obtained from the specimen, while bypassing the need to image at multiple depths and keeping the imaging time to a minimum.

The resolution of OPT depends on the size and type of specimen; however, for a 10.5 -dpc (days postcoitum) mouse embryo, it is typical to reconstruct the data into 5 - $\mu \mathrm{m}$ voxels, obtaining a resolution good enough to pinpoint individual cells if they are labeled and surrounded by unlabeled cells. In unlabeled specimens, single-cell membranes can be clearly seen.

Many of OPT's advantages derive from its being an optical technique. The fact that optical staining techniques (such as immunohistochemistry) are sophisticated enough to pinpoint tissues expressing one specific gene underlies why OPT is so suited to morphology analysis: The precise shape of an organ can be discovered without having to dissect it away from its neighboring tissue. At a more specific level, OPT also displays advantages over fluorescent optical techniques such as confocal microscopy because, in addition to handling fluorescent signals, it can also image the colored stains used in mRNA gene expression analysis.

\section{APPLICATIONS OF OPT IN DEVELOPMENTAL BIOLOGY}

From a practical point of view, two kinds of imaging role can be distinguished that highlight the value of OPT imaging: (1) providing the 3D shape of a structure or collection of structures; and (2) recording the complete distribution of a signal throughout the whole specimen. These are both evident in most of the applications listed below.

\section{Phenotyping}

One of the most important applications for OPT is to help analyze mutant phenotypes. Although the scientific community is producing mouse mutants at an increasing rate, it is widely recognized that our ability to define what has "gone wrong" at a morphological level is often inadequate. A common conclusion is that many of these mutants are, in fact, phenotypically normal, or that many genes do not have critical roles in the development of all the tissues they are expressed in. This may be true in many cases; however, we have found that the ability to generate 3D reconstructions of a particular organ relatively fast for a number of specimens (e.g., by immunohistochemistry) often highlights morphological defects that were never spotted before. A particular advantage of examining organ shapes interactively on a computer screen is that differences are much easier to spot than when examining histological sections (Sharpe et al. 2002). Features that appear insignificant on a 2D section may become obviously important once seen in the context of the surrounding 3D tissue. The result is that differences between wild-type and mutant phenotypes are easy to detect, even for researchers with little anatomical training. 


\section{Anatomical Atlases-A Deeper Understanding of Normal Development}

Vertebrate embryo development is a complex, dynamic process that involves the growth and deformation of 3D shapes over time. Although embryological studies now span more than a century of research and we know a lot about the topological connectivity of developing organs, the true 3D shape of these structures is often still not well understood. The ability to create 3D reconstructions of labeled organs relatively easily allows various areas of morphology to be more fully studied, including embryological time courses with a high temporal resolution, comparative embryology to explore phylogenetic relationships, and the variability inherent in "normal" development. A few electronic atlases have been created recently, including zebrafish development (Bryson-Richardson et al. 2007), mouse embryo development (Baldock et al. 2003), and a more focused anatomical atlas of an embryonic day 14.5 (E14.5) mouse limb (de Laurier et al. 2008). This advantage is not restricted to animals, and OPT has also been valuable in defining the 3D geometries of plant growth (Lee et al. 2006).

\section{Analyzing Gene Expression Patterns at the mRNA or Protein Level}

OPT is particularly useful for exploring the expression patterns of genes. This can be done at the RNA level (using whole-mount in situ hybridization) or at the protein level (using fluorescent immunohistochemistry). There are two big advantages of OPT for gene expression analysis. First, it gives an overview of the pattern for the whole specimen-it is therefore good for digitized databases of expression. Second, because of its relative speed (compared to traditional sectioning approaches), it is possible to analyze a significant number of specimens in a reasonable time. Examples of this include a study that compared the complete expression patterns of all 19 mouse Wnt ligands and all 10 Frizzled receptors in E11.5 mouse embryos (Summerhurst et al. 2008). Good 3D scans of at least three different stained embryos were taken for each gene (i.e., more than 60 specimens), highlighting the benefit of a mediumthroughput technique like OPT. Traditional sectioning would require the cutting of thousands of sections. As with endogenous gene expression patterns, transgenic reporter constructs can also be analyzed with OPT. Figure 2 shows the result of OPT imaging a LacZ-expressing embryo.

\section{Exploring the Distribution of Labeled Subpopulations of Cells}

Another useful function of OPT is the ability to localize labeled cells within the embryo. Figure $2 \mathrm{~B}$ shows an example of a small clone of LacZ-expressing cells in the developing brain of a mouse embryo (Wilkie et al. 2004). The high resolution clearly shows the distribution of the cells in the medial part of the developing brain. Seeing such images in their true 3D shape is important-the results from these experiments
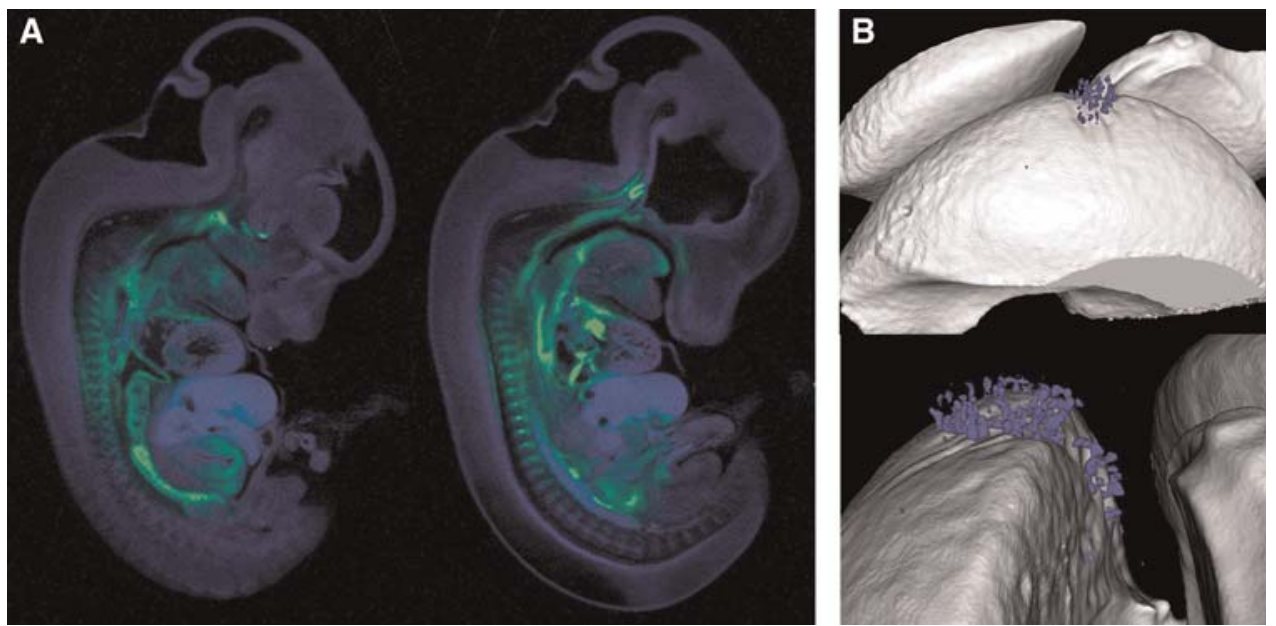

FIGURE 2. OPT imaging of LacZ-expressing tissue stained using X-gal. (A) Virtual sections through a 3D OPT image of a transgenic 12.5-dpc mouse embryo. Expression of the lacZ reporter gene (green) can clearly be localized within many internal organs of the embryo. Because lacZ was detected using X-gal (producing a blue precipitate), this signal could not be imaged by confocal. (Reproduced, with kind permission of Sharon Mulroy and Richard Sanford.) (B) 3D rendering of a small clone of LacZ-expressing cells in the developing brain of a 12.5-dpc mouse embryo (see Wilkie et al. 2004). 
were initially analyzed in 2D sections, and some of the early conclusions had to be modified upon viewing the OPT results. In general, if a colored stain is used (such as X-gal staining of the LacZ-expressing cells, shown here), small clusters of cells and, in some cases, single cells will be detectable. Another example is provided by clonal analysis in the developing mouse limb bud in a study by Arques et al. (2007). If a fluorescent dye is used (such as in fluorescent immunohistochemistry), single cells can be clearly identified.

\section{PRACTICAL ISSUES OF LABELING FOR OPT}

There are two general points to make about preparing specimens for OPT imaging. The first is that trying to image a specimen that has both a colored stain (e.g., the blue precipitate from X-gal staining) and a fluorescent signal (e.g., fluorescent immunohistochemistry) is possible but not optimal. Although it is, indeed, useful in many cases to combine fluorescent and bright-field imaging modes for a single specimen (as described below), it is not recommended to combine the two types of staining. Because the colored stain will absorb some of the light emitted from the fluorescent dye, the results produced may contain artifacts. This problem may be improved algorithmically in the future; information from the bright-field images should make it possible to estimate how much of the fluorescent light was absorbed by the colored stain.

The second point is that the optimal intensity for a good OPT scan of a colored stain is usually weaker than the best staining for visual inspection. This means that OPT is more sensitive to low-level expression, which can be an advantage; however, it also means that, when staining a specimen such as a LacZ transgenic embryo with X-gal and the staining looks good "by eye," it will often be too dark for OPT. If not enough light passes through all orientations of the embryo, the algorithm is unable to produce an accurate reconstruction. Consequently, the best results for bright-field OPT tend to be from specimens that were specifically prepared with this technique in mind.

\section{Histology/Anatomy of Unstained Specimens}

For mouse embryos $13.5 \mathrm{dpc}$ or younger, the best results are obtained using "autofluorescence." This may be due as much to the fluorescence of the fixative as to fluorescence of the tissue itself. Fluorescence images work better for small specimens because (1) fluorescence OPT generates higher-resolution images than bright-field OPT, and (2) in small unstained embryos, there tends to be insufficient optical density to absorb much light during bright-field imaging. However, as specimens become larger or more differentiated, more light is absorbed by the tissue, and optimal technique shifts away from autofluorescence imaging and toward the bright-field approach. As specimens become larger still, the choice of wavelength for bright-field illumination becomes important. Shifting toward the infrared part of the spectrum (e.g., with a wavelength up to $800 \mathrm{~nm}$ ) can significantly improve the imaging depth (see Fig. 3).

\section{Immunohistochemistry: Protein Patterns}

The optimal way to explore protein expression patterns is to use fluorescently tagged antibodies. As mentioned above, fluorescence imaging generates higher resolution than bright field, and, in addition, it appears to be impossible to "overstain" the embryo. Increasing the intensity of a colored stain, by definition, increases the opacity of the tissues, whereas increasing the strength of a fluorescent dye does not. Whole-mount immunohistochemistry can be performed on large embryos and even adult tissues. However, working with larger or more differentiated tissues tends to increase problems of background fluorescence. In some cases, this can be overcome by bleaching the tissue. Penetration of antibody staining may also be particularly problematic in certain tissues (e.g., skin) and appears to vary from antibody to antibody.

\section{In Situ Hybridization: "Gene Expression Patterns"}

The most widely used assay for whole-mount in situ hybridization (WMISH) relies on an alkaline phosphatase enzyme to catalyze the conversion of BCIP/NBT to a purple precipitate (Hammond et al. 1998). In this case, it is not the OPT imaging that limits the size of the specimen; it is the staining technique itself. Apparently, WMISH cannot penetrate all regions of an intact 12.5-dpc embryo. Because the technique is limited to embryos of $11.5 \mathrm{dpc}$ or younger, the best OPT results will come from combining bright-field imaging (for the in situ pattern) with fluorescent imaging (for the histology/anatomy of the specimen). 

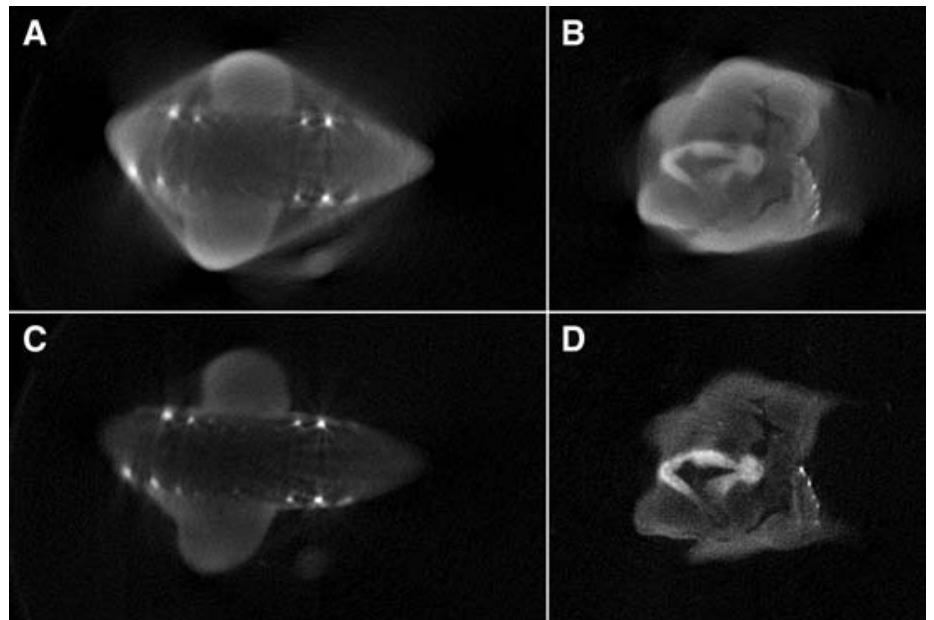

FIGURE 3. Reconstruction artifacts caused by an overstained specimen. Two virtual sections from a specimen imaged by bright-field OPT. $(A, B)$ The overstaining of this specimen means that both the signal and the background levels are too high. The appearance of the reconstruction is fuzzy, and the edges of the specimen are not clear. $(C, D)$ The best way to improve the results is to stain the specimen more weakly; however, a way to reduce the problem for specimens that have already been overstained is to use a longer wavelength. $(A, B)$ Imaged with white light; $(C, D)$ imaged with light above $700 \mathrm{~nm}$. This improves both the clarity of the tissue $(C)$ and the detection of the gene expression pattern (cf., $B, D)$.

This is similar to the LacZ results shown in Figure 2. The main complication for WMISH/OPT imaging is achieving the optimal staining level. For best results, multiple staining assays should be carried out in parallel, varying the concentration of the final substrate (or temperature/incubation time) and the staining (see Fig. 3).

\section{Transgenic Reporter Constructs}

Because LacZ staining encounters fewer penetration problems than WMISH (described above), it can be used for larger specimens, including adult organs. For smaller embryos, the best results are obtained by treating the specimen as for immunohistochemistry, using a fluorescently labeled anti- $\beta$-galactosidase antibody to benefit from the advantages of fluorescent imaging, mentioned above. However, if the X-gal method (blue precipitate) is required, good results can also be obtained, especially if the staining is not too strong. Again, several different staining intensities should be explored, and the fact that weak staining may wash out of the specimen while in BABB should be borne in mind. (In rare cases, the specimen must be imaged as soon as the BABB has equilibrated within it.) As in the case of WMISH, the best results may be achieved by combining bright-field imaging (to detect the X-gal pattern) with fluorescent imaging (to capture the histology of the specimen). However, because X-gal can work on quite large specimens (e.g., some adult mouse organs), there may be cases in which only a bright-field scan is required, as the higher opacity of the tissue will give poor results with a fluorescent scan.

Green fluorescent protein (GFP) and the other fluorescent proteins are another reporter system of great interest to many researchers. Although time-lapse imaging of these signals in living specimens has been demonstrated (Boot et al. 2008), this is currently not yet a standard procedure, whereas mapping the 3D distribution of GFP expression in fixed specimens is, in fact, very useful data for many projects. The standard GFP is not stable enough for its fluorescence to persist in alcohols; nevertheless, an easy solution is to perform whole-mount fluorescent immunohistochemistry with an antibody against the GFP protein, and this has provided excellent 3D results (e.g., de Laurier et al. 2008).

\section{Lipophilic Dyes: Tracing of Axons and Cell Movements}

Currently, tracing of axons and cell movements using lipophilic dyes is a problematic area for OPT. The achievement of high-resolution images requires a good index-matching solution such as BABB. Because these solvents disrupt lipid membranes, lipophilic dyes tend to rapidly dissolve out of the specimen. The 
discovery of alternative solvents for use with OPT, or cross-linking techniques for the dyes, could possibly overcome this limitation in the future.

\section{FUTURE PROSPECTS}

So far, OPT has proven useful in the major areas described above-that is, analysis of morphology, especially for phenotyping, and the recording of complete 3D gene expression patterns at the level of both mRNA and protein. These will probably remain the key applications in the short term; however, technological improvements may open up some new areas as well.

In particular, through improvements to the imaging mode (e.g., laser scanning instead of wide-field imaging), it may be possible to record the development of embryos or organs in culture. This would allow us to follow the dynamics of a 3D gene expression pattern as it changes during development in, for example, a mammalian embryo (using transgenic fluorescent protein reporter genes). Another development, which could contribute to this goal, is multispectral imaging, in which the intensity of fluorescently emitted light is recorded for many different wavelengths. This approach, which has been applied to other fluorescent imaging techniques, can allow multiple fluorochromes to be imaged within the same specimen.

Finally, projects such as the Edinburgh Mouse Atlas Gene Expression Database are creating a framework within which information about the spatial distribution of thousands of genes can be stored (http:// www.emouseatlas.org). This will be an invaluable resource for developmental biologists. Because OPT imaging is far more amenable to automation than traditional section-cutting approaches, it suggests for the first time that generating 3D expression data for all genes in the genome is conceivable. Such a resource would certainly be an exciting prospect for our ongoing attempts to understand developmental biology.

\section{REFERENCES}

Arques CG, Doohan R, Sharpe J, Torres M. 2007. Cell tracing reveals a dorsoventral lineage restriction plane in the mouse limb bud mesenchyme. Development 134: 3713-3722.

Baldock R, Bard J, Burger A, Burton N, Christiansen J, Feng G, Hill B, Houghton D, Kaufman M, Rao J, et al. 2003. EMAP and EMAGE: a framework for understanding spatially organized data. Neuroinformatics 1: 309-325.

Bamforth SD, Schneider JE, Bhattacharya S. 2011. High-throughput analysis of mouse embryos by magnetic resonance imaging. In Imaging in developmental biology: A laboratory manual (ed. J Sharpe, R Wong), pp. 777-787. Cold Spring Harbor Laboratory Press, Cold Spring Harbor, NY.

Boot M, Westerberg CH, Sanz-Ezquerro J, Cotterell J, Schweitzer R, Torres M, Sharpe J. 2008. In vitro whole-organ imaging: 4D quantification of growing mouse limb buds. Nat Methods 5: 609-612.

Bryson-Richardson RJ, Berger S, Schilling TF, Hall TE, Cole NJ, Gibson AJ, Sharpe J, Currie PD. 2007. FishNet: An online database of zebrafish anatomy. BMC Biol 51: 34. doi: 10.1186/1741-7007-5-34.

Davidson D, Baldock R. 2001. Bioinformatics beyond sequence: mapping gene function in the embryo. Nat Rev Genet 2: 409-417.

de Laurier A, Burton N, Bennett M, Baldock R, Davidson D, Mohun T], Logan MP. 2008. The mouse limb anatomy atlas: an interactive 3D tool for studying embryonic limb patterning. BMC Dev Biol 8: 83. doi: 10.1186/1471-213X-8-83.

Dhenain D, Ruffins S, Jacobs RE. 2001. Three-dimensional digital mouse atlas using high-resolution MRI. Dev Biol 232: 458-470.

Foster FS, Brown AS. 2011. Micro-ultrasound and its application to longitudinal studies of mouse eye development and disease. In Imaging in developmental biology: A laboratory manual (ed. J Sharpe, R Wong), pp. 669-682. Cold Spring Harbor Laboratory Press, Cold Spring Harbor, NY.

Gu S, Jenkins MW, Watanabe M, Rollins AM. 2011a. High-speed optical coherence tomography imaging of the beating avian embryonic heart. Cold Spring Harb Protoc doi: 10.1101/pdb.top98.
Gu S, Jenkins MW, Watanabe M, Rollins AM. 2011b. Optical coherence tomography imaging of early quail embryos. Cold Spring Harb Protoc doi: 10.1101/pdb.prot5564.

Hammond KL, Hanson IM, Brown AG, Lettice LA, Hill RE. 1998. Mammalian and Drosophila dachsund genes are related to the Ski proto-oncogene and are expressed in eye and limb. Mech Dev 74: 121-131.

Kak AC, Slaney M. 1988. Principles of computerized tomographic imaging. IEEE Press, New York.

Larina IV, Garcia MD, Vadakkan TJ, Larin KV, Dickinson ME. 2011. Imaging mouse embryonic cardiovascular development. In Imaging in developmental biology: A laboratory manual (ed. J Sharpe, R Wong), pp. 659-668. Cold Spring Harbor Laboratory Press, Cold Spring Harbor, NY.

Lee K, Avondo J, Morrison H, Blot L, Stark M, Sharpe J, Bangham A, Coen E. 2006. Visualizing plant development and gene expression in three dimensions using optical projection tomography. Plant Cell 18: 2145-2156.

Louie AY, Huber MM, Ahrens ET, Rothbacher U, Moats R, Jacobs RE, Fraser SE, Meade TJ. 2000. In vivo visualization of gene expression using magnetic resonance imaging. Nat Biotechnol 18: 321-325.

Massoud TF, Gambhir SS. 2003. Molecular imaging in living subjects: Seeing fundamental biological processes in a new light. Genes Dev 17: 545-580.

Metscher BD. 2011. X-ray microtomographic imaging of vertebrate embryos. In Imaging in developmental biology: A laboratory manual (ed. J Sharpe, R Wong), pp. 753-764. Cold Spring Harbor Laboratory Press, Cold Spring Harbor, NY.

Midgley PA, Weyland M. 2003. 3D electron microscopy in the physical sciences: the development of Z-contrast and EFTEM tomography. Ultramicroscopy 96: 413-431.

Mohun TJ, Weninger WJ. 2011. Episcopic three-dimensional imaging of embryos. In Imaging in developmental biology: A laboratory manual (ed. J Sharpe, R Wong), pp. 765-776. Cold Spring Harbor Laboratory Press, Cold Spring Harbor, NY. 
Ntziachristos V, Chance B. 2001. Probing physiology and molecular function using optical imaging: applications to breast cancer. Breast Cancer Res 3: 41-46.

Potter SM, Fraser SE, Pine J. 1996. The greatly reduced photodamage of 2-photon microscopy enables extended 3-dimensional timelapse imaging of living neurons. Scanning 18: 147.

Quintana L, Sharpe J. 2011. Preparation of mouse embryos for optical projection tomography imaging. Cold Spring Harb Protoc doi: $10.1101 /$ pdb.prot5639.

Ruffins SW, Jacobs RE. 2011. MRI in developmental biology and the construction of developmental atlases. Cold Spring Harb Protoc doi: $10.1101 /$ pdb.top100.

Schneider JE, Bamforth SD, Grieve SM, Clarke K, Bhattacharya S, Neubauer S. 2003. High-resolution, high-throughput magnetic paragraph sign resonance imaging of mouse embryonic paragraph sign anatomy using a fast gradient-echo sequence. MAGMA 16: 43-51.

Sharpe J. 2004. Optical projection tomography. Annu Rev Biomed Eng 6: 209-228.
Sharpe J, Ahlgren U, Perry P, Hill B, Ross A, Hecksher-Sorensen J, Baldock R, Davidson D. 2002. Optical projection tomography as a tool for 3D microscopy and gene expression studies. Science 296: 541-545.

Summerhurst K, Stark M, Sharpe J, Davidson D, Murphy P. 2008. 3D representation of Wnt and Frizzled gene expression patterns in the mouse embryo at embryonic day 11.5 (Ts19). Gene Expr Patterns 8: 331-348.

Weninger W], Mohun T. 2002. Phenotyping transgenic embryos: a rapid 3-D screening method based on episcopic fluorescence image capturing. Nat Genet 30: 59-65.

Wilkie AL, Jordan SA, Sharpe JA, Price DJ, Jackson IJ. 2004. Widespread tangential dispersion and extensive cell death during early neurogenesis in the mouse neocortex. Dev Biol 267: 109-118.

Yelbuz TM, Choma MA, Thrane L, Kirby ML, Izatt JA. 2002. Optical coherence tomography: A new high-resolution imaging technology to study cardiac development in chick embryos. Circulation 106: $2771-2774$ 


\section{Optical Projection Tomography of Vertebrate Embryo Development}

Laura Quintana and James Sharpe

Cold Spring Harb Protoc; doi: 10.1101/pdb.top116

\begin{tabular}{rc}
$\begin{array}{r}\text { Email Alerting } \\
\text { Service }\end{array}$ & Receive free email alerts when new articles cite this article - click here. \\
\hline $\begin{array}{r}\text { Subject } \\
\text { Categories }\end{array}$ & $\begin{array}{c}\text { Browse articles on similar topics from Cold Spring Harbor Protocols. } \\
\text { Developmental Biology (728 articles) } \\
\text { Imaging Development (255 articles) } \\
\text { Imaging/Microscopy, general (579 articles) } \\
\text { Visualization (524 articles) }\end{array}$ \\
\hline
\end{tabular}

